\title{
Exploiting human sensitivity to gaze for tracking the eyes
}

\author{
Nicola C. Anderson • Evan F. Risko • Alan Kingstone
}

Published online: 1 April 2011

(C) Psychonomic Society, Inc. 2011

\begin{abstract}
Given the prevalence, quality, and low cost of web cameras, along with the remarkable human sensitivity to gaze, we examined the accuracy of eye tracking using only a web camera. Participants were shown webcamera recordings of a person's eyes moving $1^{\circ}, 2^{\circ}$, or $3^{\circ}$ of visual angle in one of eight radial directions (north, northeast, east, southeast, etc.), or no eye movement occurred at all. Observers judged whether an eye movement was made and, if so, its direction. Our findings demonstrate that for all saccades of any size or direction, observers can detect and discriminate eye movements significantly better than chance. Critically, the larger the saccade, the better the judgments, so that for eye movements of $3^{\circ}$, people can tell whether an eye movement occurred, and where it was going, at about $90 \%$ or better. This simple methodology of using a web camera and looking for eye movements offers researchers a simple, reliable, and cost-effective research tool that can be applied effectively both in studies where it is important that participants maintain central fixation (e.g., covert attention investigations) and in those where they are free or required to move their eyes (e.g., visual search).
\end{abstract}

Keywords Eye tracking $\cdot$ Web camera $\cdot$ Gaze following

\author{
N. C. Anderson $(\bowtie) \cdot$ A. Kingstone \\ Department of Psychology, University of British Columbia, \\ 2136 West Mall, \\ Vancouver, BC, Canada V6T 1Z4 \\ e-mail: nccanderson@psych.ubc.ca \\ E. F. Risko \\ Social and Behavioral Sciences Department, \\ Arizona State University, \\ Glendale, AZ, USA
}

Eye-tracking technology has been used in fields of research as diverse as neuroscience (Findlay \& Walker, 1999; Snodderly, Kagan, \& Gur, 2001), psychology (Birmingham, Bischof, \& Kingstone, 2008; Henderson \& Hollingworth, 1998; Rayner, 1998), aviation (Anders, 2001), driving (Land \& Lee, 1994), marketing (Wedel \& Pieters, 2000), and a wealth of others (for reviews, see Duchowski, 2002; Richardson \& Spivey, 2004). Despite its widespread use and increasing sophistication, eyetracking also carries some significant disadvantages. For example, it is expensive, requires time-consuming calibrations, and is difficult, if not impossible to use covertly. The latter consideration has recently been brought into focus by the demonstration that people will change the way that they respond to their environment when they know that their eyes are being monitored (Risko \& Kingstone, 2011). For instance, Risko and Kingstone discovered that people avoid looking at a provocative stimulus (a sexy swimsuit calendar) if they believe that the eye monitor they are wearing is turned on (they readily look at the swimsuit calendar if they believe that the monitor is turned off). What is needed is a simple, inexpensive way to discretely and reliably monitor people's eyes.

The purpose of the present investigation is to introduce such a method. Spring-boarding from the fact that it is now possible to record video with high-definition clarity using a web camera that is literally a thousand-fold cheaper than a research eye monitor, we recorded the eyes of people making small eye movements of $1^{\circ}, 2^{\circ}$, or $3^{\circ}$ of visual angle in any one of eight different directions (north, northeast, east, southeast, etc.), or no eye movement occurred at all. Then, capitalizing on established findings that humans are remarkably sensitive to where other people are looking (Anstis, Mayhew, \& Morley, 1969; Bock, Dicke, \& Their, 2008; Cline, 1967; Gibson \& Pick, 1963; Kobayashi \& Kohshima, 2001; Symons, Lee, Cedrone, \& Nishimura, 2004), we hypothesized that one could record eye movements on a 
web camera and present them to human observers who would then determine reliably and with a high degree of fidelity whether an eye movement occurred and where it was directed.

Broadly speaking, studies concerned with the eye movements of human participants can be placed in one of two categories: (1) investigations that seek to ensure that participants are not making eye movements, as is typically the case in studies of human covert attention (e.g., cuing studies, Gibson \& Kingstone, 2006; attentional capture, Schreij, Owens, \& Theeuwes, 2008; and word naming, Risko, Stolz, \& Besner, 2010) and (2) studies that permit eye movements and are focused on using where an individual looks to make inferences about underlying mental processes (e.g., visual search, Wolfe, 2010; oculomotor capture, Theeuwes, Kramer, Hahn, \& Irwin, 1998; and preferential looking, Fernald, Zangl, Protillo, \& Marchman, 2008). The present research will provide important information regarding both of these functions of eye tracking. That is, our findings regarding the ability of people to detect eye movements recorded via a webcamera are of particular relevance to those researchers who are interested in using our research method in studies that instruct participants to avoid making an eye movement. Here, our findings will speak to the extent that one can be confident that any eye movement of a particular size and direction will be detected. In addition, our findings regarding the ability of people to use a web camera to determine the direction of recorded eye movements are relevant for researchers interested in employing such a system for determining where an individual is looking.

To anticipate our results, we demonstrate that by using just a web camera and a human observer, one can detect and discriminate the location of eye movements as small as $1^{\circ}$ of visual angle. Critically, as the visual angle of these eye movements increases, so does the ability to detect and discriminate them, such that, when eye movements of merely $3^{\circ}$ are executed, the detection of any eye movement, regardless of its direction, is greater than $90 \%$. For eye movements in the cardinal directions alone (north, south, east, and west), the discrimination accuracy is similarly high. Thus, if one wants to know if and/or where participants are looking, simply placing a target item $3^{\circ}$ or more away from fixation will be sufficient. Note that most covert attention paradigms already place targets at eccentricities greater than $3^{\circ}$, so our methodology can readily be applied in studies where maintaining central eye fixation is important. Similarly, for studies that require knowing precisely where a participant has looked, an item placed at just $3^{\circ}$ will lead to a similar level of accuracy in identification of a participant's eye movement to a target.

In all of these research domains, the web camera plus human observer system provides the benefits of being inexpensive, nonintrusive, and able to track blinks and offscreen fixations. It has the additional benefit of providing the researcher with responses that include the entire face, such as emotional changes (Brennan, Watson, Kingstone, \& Enns, 2010) and fluctuations in alertness and mindwandering (Chisholm, Risko, \& Kingstone, 2010), responses that cannot be observed directly from an expensive eye monitor.

In the following work, we describe first how the web camera stimuli are recorded and presented to coders, then we provide an analysis of the human-coded eye movements that have implications for the practical use of this method in current behavioral research.

\section{Method}

Stimulus generation phase

We recorded a total of 1,512 gaze stimuli from four models ( 2 men, 25 and 24 years old, and 2 women, 24 and 27 years old), of which 1,344 contained an eye movement in one of eight radial directions at three possible distances $\left(1^{\circ}, 2^{\circ}\right.$, or $3^{\circ}$ of visual angle) and 168 videos of the same models not making an eye movement (i.e., fixating the center of a display). The entire database of eye movements is available to researchers through contacting the corresponding author (nccanderson@psych.ubc.ca).

To create the gaze stimuli, each model was placed in a chinrest and was asked to make eye movements in response to targets that were presented on a Dell 2407WFP 17-in. (diagonal) monitor located approximately $60 \mathrm{~cm}$ in front of the model. An SR Research Eyelink 1000 was used in conjunction with SR Research Experiment Builder to present the stimuli and verify the eye movements made. Eye gaze position was tracked by pupil and corneal reflection and was sampled at a rate of $1000 \mathrm{~Hz}$. Before beginning, a 9-point calibration and validation was conducted. The web camera was centered at the top of the monitor and angled slightly downward to record videos of the model's gaze. The web camera's native software was used to position and zoom the camera such that each person's head covered roughly $10 \mathrm{~cm}$ of the video window.

Each model participated in two sessions of eye movement recording in order to generate stimuli at two different web camera resolutions: one in which the camera was set to encode at $640 \times 480$ pixels at a frame rate of 15 frames per second (fps), hereafter referred to as the low-resolution condition, and one in which the camera was set to encode at $960 \times 720$ pixels at a frame rate of $15 \mathrm{fps}$, hereafter referred to as the high-resolution condition. Sessions were counterbalanced across gender, such that one female and one male started with the high-resolution session and the other male 
and female started with the low-resolution session. Each session lasted approximately $1 \mathrm{~h}$. The high-resolution video clips were resized to $640 \times 480$ pixels. Thus, they were identical in size to the low-resolution videos but contained more pixels per inch and were subjectively more clear and detailed.

Each individual "trial" consisted of the presentation of a central fixation cross in conjunction with a tone that was used to demarcate the beginning of each trial. The model was required to fixate the cross for up to $1,000 \mathrm{~ms}$, after which a circular target (12 pixels in diameter, subtending $0.68^{\circ}$ of visual angle) would appear in one of the eight radial directions and at one of the three distances, as described above. The model was then instructed to move his or her eyes to this circular target. In the no-eyemovement condition, the central fixation cross was replaced by the target. To ensure that the model's eye movements corresponded to the correct distance and direction (within the limits of the Eyelink 1000's eye-tracking capabilities), each target was surrounded by an invisible interest area (circular, 32 pixels in diameter, subtending $1.8^{\circ}$ of visual angle), and the model was required to accurately fixate within this interest area before the trial would terminate. Each of the possible 24 eye movement locations ( 8 directions $\times 3$ distances) was repeated 7 times throughout stimulus generation, with 3 no-eye-movement trials for each of the three targets that would appear in any one direction, for a total of 189 trials: 168 eye movement trials and 21 no-eye-movement trials. Trials were randomized with breaks and recalibrations as needed throughout.

These videos were then manually cut into 1.5 long segments containing the gaze stimuli using FFmpeg (Bellard, 2007), an open-source video capture and encoding software published under the GNU public license. The tone that occurred in conjunction with the central fixation during stimulus generation was used as a reference for the beginning of the trial. Thus, a typical clip would show the person looking at the fixation point and then executing an eye movement or not (depending on the particular trial). Clips did not show the person moving his or her eyes back to the central fixation cross.

\section{Experimental phase}

Participants Eight participants (4 men and 4 women) volunteered to participate in the experimental phase. Presentation order was counterbalanced such that half saw the high-resolution videos first and the other half saw the low-resolution videos first.

Stimuli Stimuli were the 1,512 web camera video clips from the stimulus generation phase described above. The SR Research Experiment Builder was used to display the stimuli.
In order to load the videos into Experiment Builder, they needed to be passed through the DivX codec (DivX Inc, San Diego, CA) and have their audio removed. SR Research's SplitAVI tool was used to batch convert and remove the audio of all the video files. Excess space around the clip was colored black. On average, the horizontal angular resolution of the models' heads was $9.76^{\circ}$ of visual angle.

Apparatus Stimuli were presented on a Dell 2407WFP 17in. monitor with a resolution of $1,024 \times 768$ pixels. Participants were instructed to sit comfortably and adjust the chair such that their eyes were slightly higher than the middle of the monitor, which was $60 \mathrm{~cm}$ away.

Procedure Participants were instructed that on each trial, they would be presented with a video clip and that they had to decide the direction in which an eye movement had been made or whether no eye movement had been made. Each trial consisted of one video clip presented for its entire duration (i.e., $1.5 \mathrm{~s}$ ). After the video clip ended, the last frame of the clip remained visible on the screen until a response had been made or $10 \mathrm{~s}$ had elapsed. Participants responded to the direction of the eye movement that they perceived using the number pad. Each number on the number pad corresponded to one of the possible radial directions presented in the clip. Thus, if participants perceived that the eye moved up and to the left, they would respond using the " 7 " key on the number pad. If they perceived the eye to be moving down and to the right, they would respond using the " 3 " key on the number pad, and so forth. If participants did not perceive an eye movement at all, they would press the " 5 " key on the number pad. After the participants had responded, a blank screen was presented, and participants pressed the spacebar to proceed to the next trial.

Each participant completed two separate testing sessions counterbalanced across resolution. Within each session, trials were randomly blocked by model, such that participants would respond to all 189 gaze clips of one model, then move on to the next, and so forth. Each session lasted approximately $45 \mathrm{~min}$.

\section{Results}

Responses were broken down into two components: detection and discrimination. Correct detection occurred if the participant responded that the eye had moved on eye movement trials, regardless of whether they were correct in their direction assessment. Direction discrimination was defined as the number of eye movements at each distance and direction that were correctly discriminated (i.e., 
participants responded with the correct direction) out of the number of eye movements at each distance and direction that were correctly detected. These two measures, detection and discrimination, were analyzed separately, and the results are presented below. Figures 1 and 2 show the proportion correctly detected and discriminated, respectively, for each distance and at each direction.

Detection A 2 (resolution) $\times 3$ (distance) $\times 8$ (direction) repeated measures analysis of variance was conducted on the proportion of eye movements that were correctly detected. There was a marginal main effect of resolution, $F(1,7)=$ $3.79, M S E=0.11, p=.09$, such that eye movement detection was better for the high-resolution videos (.72) than for the low-resolution videos (.69). There was a main effect of distance, $F(2,14)=266.31, M S E=0.27, p<.001$, such that $2^{\circ}$ eye movements (.79) were more likely to be detected than $1^{\circ}$ eye movements $(.41), t(7)=28.5, p<.001$, and $3^{\circ}$ eye movements (.92) were more likely to be detected than $2^{\circ}$ eye movements, $t(7)=5.52, p<.001$. In addition, the difference in detection between $1^{\circ}$ and $2^{\circ}$ eye movements (.38) was significantly larger than the difference in detection between $2^{\circ}$ and $3^{\circ}$ eye movements (.12), $t(7)=10.11, p<.001$. There was also a main effect of direction, $F(7,49)=19.54, M S E=$ $0.27, p<.001$. Inspection of the mean detection rates at each direction showed an advantage for upward eye movements (northeast, north, and northwest), and a paired samples $t$-test confirmed that these eye movements (.80) were significantly easier to detect than the eye movements in all other directions combined (.67), $t(7)=8.81, p<.0001$.

There was a significant interaction between resolution and distance, $F(2,14)=13.44, M S E=0.06, p<.001$. Paired $t$-tests comparing the effect of resolution (high-low) at the three distances revealed that the effect of resolution decreased with increases in the size of the eye movement. Specifically, the effect of resolution at a distance of $1^{\circ}(.08)$ was marginally larger than the effect of resolution at $2^{\circ}$ $(.03), t(7)=2.14, p=.07$, and the effect of resolution at $2^{\circ}$ was significantly larger than the effect of resolution at $3^{\circ}$ $(-.01), t(7)=3.01, p<.05$. Only the effect of resolution in the $1^{\circ}$ eye movement condition was significantly different from $0, t(7)=6.32, p<.001$.

There was a marginally significant interaction between resolution and direction, $F(7,49)=2.01, M S E=0.01 p=$ .072. Inspection of the means suggested a large effect of resolution in the straight downward direction, and in fact, the effect of resolution (high-low) on detection of straight downward eye movements (.09) was significantly greater than the effect of resolution inall other directions $(.03), t(7)=$ $4.89, p<.002$.

There was a significant interaction between distance and direction, $F(14,98)=8.43, M S E=0.06, p<.001$. In order to interpret this interaction, the slope relating the size of the eye movement to detection was calculated for each participant at each direction. Thus, shallower slopes indicate a smaller effect of eye movement size on detection. Visual inspection of the distance by direction slopes revealed that eye movement size had less of an effect on the upward eye movements. A paired samples $t$-test confirmed that the average slope of the upward directions (northwest, north, northeast; .19) was significantly shallower than the average slope of the other directions combined (.29), $t(7)=5.724, p<.001$.

Lastly, there was a significant resolution $\times$ distance $\times$ direction interaction, $F(14,98)=2.88, M S E=0.01, p<$ .002 . The slopes relating the distance the eye moved to detection were again calculated, this time for each participant at each direction and resolution. The interaction
Fig. 1 Proportions of correctly detected eye movements. Error bars represent $95 \%$ confidence intervals based on the mean square error of the highest level interaction (Masson \& Loftus, 2003)

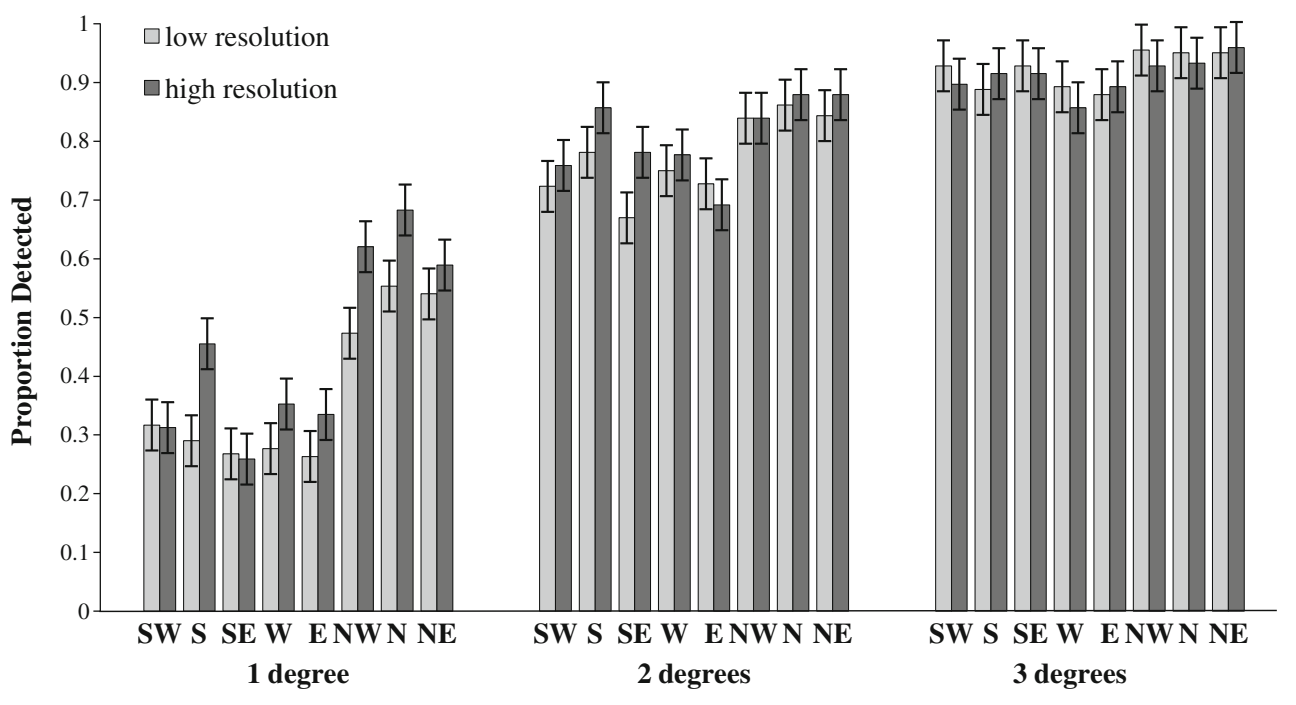


Fig. 2 Proportions of correctly discriminated eye movements. Error bars represent 95\% confidence intervals based on the mean square error of the highest level interaction (Masson \& Loftus, 2003)

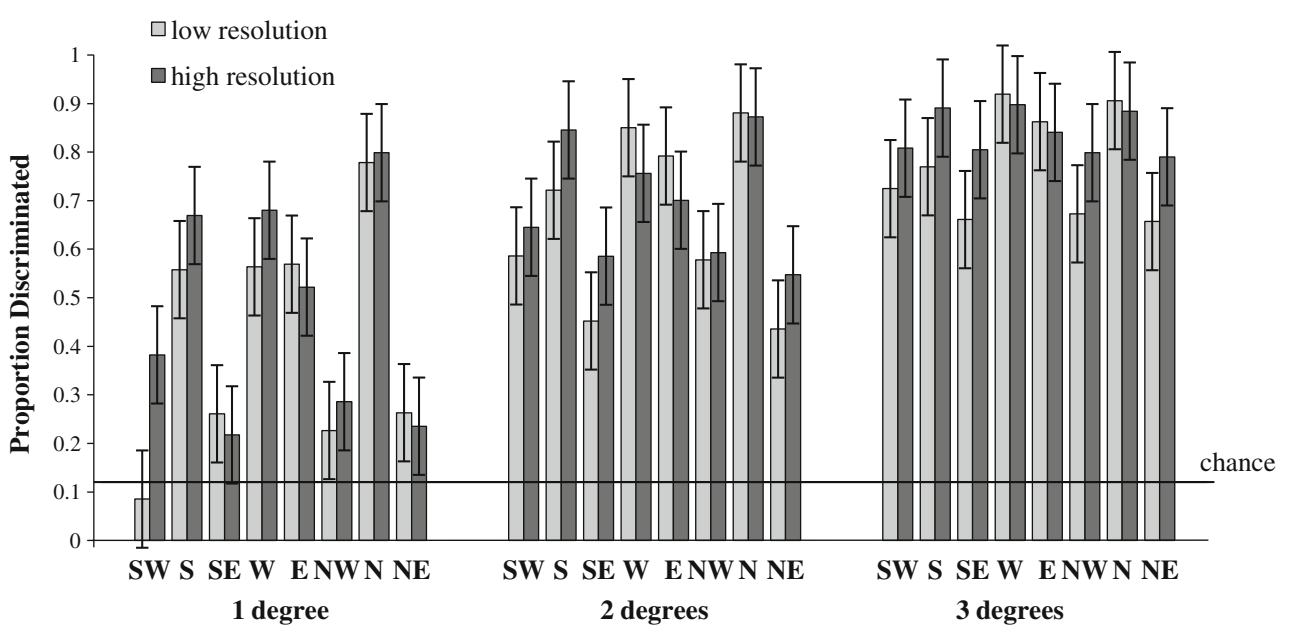

appears to result from the fact that increasing resolution reduces the effect of distance, but only in certain directions: south (slope difference, .07), $t(7)=3.61, p<.01$, northwest (slope difference, .09), $t(7)=8.96, p<.001$, and north (slope difference, .07), $t(7)=3.56, p<.01$.

False alarms A paired samples $t$-test was conducted on the proportion of false alarms on no-eye-movement trials (i.e., the participant indicated that an eye movement had been made when it had not). False alarms were rare (.12), and a paired samples $t$-test revealed that there was no significant difference between high- (.125) and low- (.121) resolution trials, $t(7)=0.166, p=.873$.

Signal detection We also conducted a signal detection analysis. A measure of sensitivity, $d^{\prime}$, was calculated for each participant across each resolution, distance, and direction. In order to account for cases where the hit rate was $100 \%$ and $d^{\prime}$ took on an unlimited value, hit rate proportions of one were converted as suggested by Macmillan and Creelman (1991) to $[1-1 /(2 N)]$. $d^{\prime}$ values were submitted to a 2 (resolution) $\times 3$ (distance) $\times 8$ (direction) repeated measures analysis of variance. The pattern of results was similar, as might be expected, to the detection results above; however, the marginal main effect of resolution (in the detection analysis) disappeared, $F(1,7)=0.245, M S E=0.723, p=.636$. The only other difference in this analysis was in the resolution $x$ distance interaction, $F(2,14)=6.74, M S E=.706, p<.01$. The effect of resolution at $1^{\circ}(.20)$ was no longer marginally different from the effect of resolution at $2^{\circ}(.14), t(7)=0.606$, $p=.564$, and in addition, it was no longer significantly different from $0, t(7)=1.24, p=.256$. Nonetheless, the pattern of results using $d^{\prime}$ was largely equivalent to the detection results. Critically, the $d^{\prime}$ values at $1^{\circ}$ (1.04) were significantly different from zero in a single-sample hypothesis test, $t(7)=17.91, p<.001$, indicating that viewers were indeed sensitive to these small eye movements, over and above chance alone.

Summary for correct detection and false alarms In summary, detection was better for larger eye movements and for eye movements in the upward directions. The size of the eye movement had less effect on the detection of upward eye movements than on those in the other directions. Resolution improved the detection of $1^{\circ}$ eye movements in general and also improved detection of straight downward eye movements but had no effect on the proportion of false alarms, which were relatively infrequent. Sensitivity measures closely matched those of the detection results but also revealed a significant advantage of the $1^{\circ}$ eye movements over chance. In the next section, direction discrimination while holding eye movement detection constant is assessed.

Discrimination Correct discrimination was calculated as the number of correct responses divided by the total number of eye movements that were detected in each direction and at each distance. This provided a discrimination value that was corrected for detection. A 2 (resolution) $\times 3$ (distance) $\times$ 8 (direction) repeated measures analysis of variance was conducted on these values. There was a main effect of distance, $F(2,14)=180.23, M S E=4.30, p<.001$, such that responses were significantly more accurate for $2^{\circ}$ eye movements (.68) than for $1^{\circ}$ eye movements $(.44), t(7)=$ $10.82, p<.0001$, and were significantly more accurate for $3^{\circ}$ eye movements $(.81)$ than for $2^{\circ}$ eye movements, $t(7)=$ $15.07, p<.0001$. In addition, the difference in discrimination between $1^{\circ}$ and $2^{\circ}$ eye movements (.24) was significantly greater than the difference in discrimination between $2^{\circ}$ and $3^{\circ}$ eye movements $(.13), t(7)=4.86, p<.005$. There was a main effect of direction, $F(7,49)=21.60, M S E=1.01, p<$ .0001 . Inspection of the direction means revealed an advantage for the cardinal directions (north, east, south, 
west) over the oblique directions (northwest, northeast, southwest, southeast), and averaging accuracy into cardinal and oblique groups showed a significant advantage in terms of accuracy for cardinal (.77) relative to oblique (.51) directions, $t(7)=9.32, p<.0001$.

There was a significant interaction between resolution and direction, $F(7,49)=5.31, M S E=0.05, p<.001$. Inspection of the means indicated that resolution had less effect on the cardinal than on the oblique directions. Again, means were grouped into cardinal and oblique directions, and the effect of resolution was significantly higher in obliques (.09) than in cardinals (.02), $t(7)=3.30, p<.02$. In addition, single-sample hypothesis tests showed that the effect of resolution on oblique directions was significantly different from $0, t(7)=3.57, p<.01$, and that the effect of resolution on the cardinal directions was not significantly different from $0, t(7)=0.388, p=.709$.

There was a significant interaction between distance and direction, $F(14,98)=8.52, M S E=0.10, p<.0001$. The slope relating the size of the eye movement to discrimination was calculated for each participant at each direction, shallower slopes indicating a smaller effect of eye movement size on discrimination. Again, grouping slope scores into cardinal and oblique directions showed that cardinals (slope average, .12) were less affected by distance than were obliques (slope average, .25), $t(7)=6.69, p<.001$. No other significant interactions were found.

Summary for direction discrimination Direction discrimination improved with the size of the eye movement, and this improvement was more pronounced between $1^{\circ}$ and $2^{\circ}$ eye movements than between $2^{\circ}$ and $3^{\circ}$ eye movements. Cardinal eye movements were better discriminated than oblique eye movements, and discrimination of cardinal eye movements was less affected by the size of the eye movement than was discrimination of the obliques. Lastly, resolution improved discrimination of the oblique eye movements, but not eye movements in the cardinal directions.

Model analysis We conducted a 2 (resolution) $\times 3$ (distance) $\times 4$ (model) $\times 8$ (direction) analysis of variance on both the detection and discrimination results. In the detection results, there was a significant resolution $\times$ model interaction, $F(3,21)=8.85, M S E=.399, p<.002$, a distance $\times$ model interaction, $F(6,42)=6.74, M S E=.195, p<.0001$, and model $\times$ direction interaction, $F(21,147)=5.22, M S E=$ $.111, p<.0001$. In addition, there was a significant threeway interaction between distance, model, and direction, $F$ $(42,294)=2.36, M S E=.039, p<.0001$. All other interactions were the same as in the previous detection results. In the discrimination results, there was a significant main effect of model, $F(3,21)=13.37, M S E=.802, p<.0001$, a significant resolution $\times$ model interaction, $F(3,21)=5.03$, $M S E=.355, p<.01$, a significant distance $\times$ model interaction, $F(6,42)=2.46, M S E=.153, p<05$, a significant model $\times$ direction interaction, $F(21,147)=3.15, M S E=$ $.214, p<.0001$, a significant resolution $\times$ model $\times$ direction interaction, $F(21,147)=1.75, M S E=.095, p<.05$, and a significant distance $\times$ model $\times$ direction interaction, $F(42$, 294) $=2.37, M S E=.103, p<.0001$. All other interactions were the same as those in the previous discrimination results. Inspection of the means while breaking down each of these effects and interactions suggested that the model's gender may have influenced the detection and discrimination of the eye movements. We report the previous analysis to support the notion that accuracy of eye movement detection and discrimination will vary with model, as can be expected given variation in eye shape, pupil size, and so forth (this is true of all eye-tracking techniques). We do not attempt to break down or provide explanations for these interactions, given that (1) there were only four models and (2) any differences were likely idiosyncratic to the models we used and, thus, of little value to researchers. However, the idea of individual differences in eye movement discriminability certainly warrants further investigation, if for no other reason than the potential evolutionary consequences of variation in this aspect of our physical makeup (Kobayashi \& Kohshima, 2001). Figure 3 provides a breakdown of the detection and discrimination means for each model.

\section{Discussion}

In the present investigation, we sought to determine the potential utility of a web camera plus human observer system for the detection and discrimination of eye movements. The results were both impressive and informative.

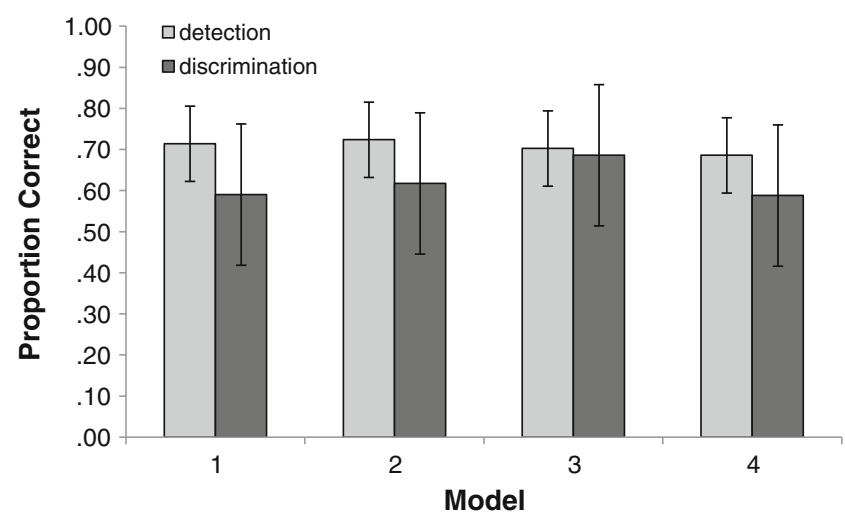

Fig. 3 Proportion correctly detected or discriminated for each model. Models 2 and 3 were female. Error bars represent 95\% confidence intervals based on the mean square error of the highest level interactions (Masson \& Loftus, 2003) 
Eye movements as small as $1^{\circ}$ of visual angle could be detected and discriminated by human coders with a high degree of fidelity, and once a saccade was a mere $3^{\circ}$, detection was over $90 \%$ and correct discrimination over $80 \%$. In addition, detection and discrimination values improved across distance in all directions, while directional effects revealed that eye movements in the upward directions were particularly noticeable to coders and eye movements in the cardinal directions were better discriminated than those in the oblique directions. Thus, the web camera provides a cheap and effective way of monitoring whether people make an eye movement and where that eye movement is directed. In addition, the results suggest a number of important considerations when such a system is employed.

Overall, the results indicate that the proposed research method of using a web camera to monitor eye movements and a human observer to judge them is an extremely effective tool both for studies that put a priority on participants' maintaining fixations and for those that allow or require eye movements. In the following, we discuss the potential applications of the present research method for monitoring eye movements, provide practical suggestions for calibrating such a system, and briefly discuss the limitations of the proposed research method, as well as its relation to current technology and previous work.

Utility of the system A significant advantage to using a web camera to detect eye movements is that it can be used discretely, without any calibration. This is particularly important for researchers wishing to examine the role of the larger social context on visual behavior - specifically, in attenuating the behavioral changes that occur when participants know, as in every eye-tracking experiment, that their eyes are being monitored (e.g., Risko \& Kingstone, 2011). In general, however, this method can be particularly useful for those who are focusing on a more ethological approach (Hutchins, 1995; Kingstone, Smilek, \& Eastwood, 2008), since an unexplored advantage to this method may be the ability to simultaneously record facial expressions, speech, body posture, head turns, and other behavioral measures, in addition to eye movements (Brennan et al., 2010). It is important to keep in mind, however, the ethical considerations that such unobtrusive eye monitoring engenders.

One potential application of the web camera plus human observer system is for studies that require the maintenance of central fixation-for example, the covert orienting experiments introduced by Posner (1980). In this paradigm, individuals are instructed to maintain gaze on a fixation point while responding to targets in the periphery. Critically, in order to draw inferences about covert attention (i.e., shifts of attention without an eye movement), it is imperative that the participants maintain fixation. However, eyetrackers are rarely used to check whether participants are complying with this instruction (e.g., Pratt, Kingstone, \& Khoe, 1997; Risko et al., 2010), which can have a profound effect on the results that are obtained (Kingstone \& Pratt, 1999). A web camera plus human observer system could be used to detect illicit eye movements in this case or any case where illicit eye movements would need to be detected. The present results suggest that the utility of our particular method would be directly proportional to the size and direction of the eye movements made (i.e., the system will be better able to detect large illicit eye movements). Given that detection of small eye movements was easier in the upward directions, it may be beneficial to place not-tobe-fixated stimuli toward the top of the screen, thus making it easier to detect whether an eye movement to that stimulus is made. Another critical observation made here that supports the use of a web camera plus human observer system in the context of detecting illicit eye movements is the significant sensitivity to $1^{\circ}$ eye movements.

The web camera plus human observer system will also be relevant for domains wishing to explore intentional eye movements - essentially, any experiment using the preferential looking paradigm (Fantz, 1965; for examples, see Fernald et al., 2008; Spelke, Breinlinger, Macomber, \& Jacobson, 1992) - or where objects or scenes are welldefined, arranged side by side, or in an array. The present work suggests that targets in such experiments should be placed along the horizontal and vertical axis where possible, to take advantage of the increased accuracy in the discrimination of eye movements in the cardinal directions. In addition, our work suggests that the farther apart the targets are, the easier it will be to determine which target is being looked at by the participant, although a mere $3^{\circ}$ of visual angle is sufficient to trigger excellent discrimination accuracy. Many preferential looking paradigms already use video to increase the temporal resolution of their results (see Fernald et al., 2008). Our results further corroborate this paradigm's utility. They also provide a general guideline for where targets could be placed in order to increase the perceptibility of the eye movements to the human coders, thereby increasing the reliability of the results.

Eye movements sampled in such a noninvasive way may also be useful for those researchers concerned with special populations where traditional head-worn eye-tracking equipment is undesirable and where calibration may be difficult. For example, patients with visual neglect often find it difficult, if not impossible, to saccade toward or maintain fixation in contralesional visual space. Traditional eye-tracking equipment does not operate under such circumstances, requiring calibration across the entire tracking area before a trial can begin, and often, special equipment and procedures are needed in order to calibrate visual neglect patients (Dunai, 
Bennett, Fotiades, Kritikos, \& Castiello, 1999). Our human coders did not need any such calibration in order to see an eye movement.

Improving the system While the present results bode well for the human observer plus web camera system, there is room for improvement. For example, it is possible that participants simply did not see the smallest eye movements, since iris deviations were very near the levels of visual acuity; however, this is unlikely given the directional effects we observed in these eye movements. It may be beneficial to zoom the camera in toward the eyes as much as possible during recording or have the participant sit closer to the web camera, thereby maximizing the apparent size of the eye movement and increasing the movement of the iris well above acuity levels. However, this particular method is unlikely to be useful for the detection of very small drifts of the eye, tremors, or microsaccades, since it is unlikely that current off-the-shelf web cameras have the optical or temporal resolution to pick up on these aspects of natural fixation (Martinez-Conde, Macknik, \& Hubel, 2004).

The directional effects in our results indicate that limiting the number of targets might improve discrimination accuracy. For example, if one were concerned with detecting or discriminating upward and downward eye movements (or those to the left and right), presumably, detection and discrimination accuracy would increase as possible target locations decreased. Inspection of the stimulus videos in the present work and subjective report revealed that eyelid movement was especially predominant in the upward eye movement stimuli, something that we suspect was involved in the overall $80 \%$ detection rate of these eye movements. Thus, eyelid movement may provide a broad additional cue to the movement of the eyes that can be capitalized on by limiting target locations.

The Logitech Webcam Pro 9000 has the ability to record low-resolution video $(640 \times 480)$ at $30 \mathrm{fps}$. The saccades in this experiment were expected to last less than $50 \mathrm{msec}$ (Leigh \& Zee, 2006), and since each frame in our 15 fps video capture lasted $66.7 \mathrm{msec}$, it is possible that saccades occurred between frames. Thus, there is reason to believe that recording at $30 \mathrm{fps}$, which would potentially capture a saccade in two or more frames, might improve detection and discrimination by lengthening the captured saccadic duration.

Accurate detection and discrimination of eye movements is limited only by the quality of the video and the visual system of the coder. Detection and discrimination seem to be dependent on minute changes in the stimuli, such as the sclera area to iris ratio (Anstis et al., 1969) and, possibly, eyelid movement. Given that the detection of the smallest eye movements and the discrimination of the obliques significantly improved in the high-resolution condition of our experiment, it may be beneficial to obtain the best possible camera for the most robust results, especially considering that the average cost of a web camera is several orders of magnitude less than the average cost of an eyetracker. In addition, the position of the camera may have exaggerated the upward eyelid movement in our experiment. It is possible that this exaggeration is responsible for the higher detection and discrimination rates of these upward eye movements; thus, the positioning of the camera may be of vital importance in helping coders to see these eye movements.In our experiment, each participant was allowed to see a unique eye movement stimulus only once. If participant coders in our work had been given the opportunity to replay the web camera recordings, perhaps the already excellent detection and discrimination rates would have been further improved. Furthermore, in a practical setting, judgments from multiple coders could be compared and integrated in order to decide whether an eye movement had been made and/or which direction it went in a given video segment.

Limitations While we have emphasized the potential utility of the system in various contexts, it is also important to note explicitly the contexts that are not appropriate for such a system. First, the method assessed is purely an offline system; thus, any online eye tracking (e.g., gaze-contingent designs, trial-by-trial performance feedback, etc.) is not advisable. In addition, our results suggest that eye movements to crowded stimuli, such as those used in certain visual search paradigms, would be extremely difficult to dissociate. The utility of the system will naturally depend on the nature of the experimental design used in conjunction with it. It is important to note that we are not suggesting that the web camera plus human observer system can replace commercially available eyetrackers in all contexts; rather, we would like to argue, and the results of the present study support, that there exist contexts (possibly many) where the former system can be put to use in lieu of the more expensive options. The results of the present study will allow researchers to make the discrimination between contexts that are appropriate for the use of the web camera plus human observer system.

Relation to current technology The chief concern of this article was to highlight a methodology of gaze tracking with web cameras and human observers that is simple to use, inexpensive, accurate, and reliable. The common alternative is typically to use commercial eyetrackers and software. The best accuracy reported for the SR Research Eyelink 1000 system is $0.5^{\circ}$ of visual angle (SR Research), provided that head movement is kept to a minimum and that certain lighting conditions are met. The Tobii X120, which is often used to track the eyes of populations where restricting head motion is not possible, advertises an accuracy as good as $0.25^{\circ}$ of visual 
angle. In practice, these values are an upper bound on the actual accuracy typically obtained with these systems. They are, nevertheless, extremely effective systems for online, high-precision eye tracking. However, they do not come cheap, with a price tag upward of $\$ 45,000$ (USD). This price does not include the training and expertise required to operate and maintain such systems.

The use of a web camera to track the eyes in not a new idea (e.g., Pedersen \& Spivey, 2006), and the image processing and machine vision research required to overcome some of the problems with web camera gaze tracking represents an active area of research (Hansen \& Pece, 2005). There are many algorithms that exist to track gaze with web camera input (e.g., Hansen \& Pece, 2005; Vivero, Barreira, Penedo, Cabrero, \& Remeseiro, 2010; Wang \& Sung, 2001), some of which are open source (Li \& Parkhurst, 2006; Pedersen \& Spivey, 2006). Most of these systems are geared toward real-time eye tracking and, as such, are not yet nearly as accurate as typical commercial systems. The open-source programs can be used with both infrared web cameras and visible spectrum cameras but require extensive calibration for proper tracking.

All of the systems above differ from the proposed system in one critical area: the human observer. While many of the systems above use webcameras, the aim is to automate the detection of eye movements. This automation requires the introduction of restrictions (e.g., the need for calibration) not present when a human observer plays the equivalent role. The web camera plus human observer system also has restrictions, although the restrictions are, of course, different in nature (i.e., you need a human observer). The assessment of the combination of web camera and human observer represents the unique contribution of the present work (not the use of web cameras for eye tracking). Together, the development of each of these different strategies (e.g., web camera plus software tracking, web camera plus human observer) and their judicious application in research on eye tracking promise to open this method to a much wider audience.

Relation to previous work Our results corroborate the only other previous work exploring the accuracy of human gaze detection and discrimination that examined eye movements to targets other than those in the horizontal and vertical planes. Bock et al. (2008) showed an upward and cardinal direction bias in eye movement discrimination at $15^{\circ}$ eccentricity in a dynamic video condition. Although we found only an upward bias in the detection results and a cardinal bias in the discrimination results, our work loosely extends Bock et al.'s (2008) findings to include eye movements at profoundly smaller eccentricities as well.

One common theme in the psychophysical gazefollowing literature is that without proper stimulus control, it is difficult to make conclusions about the specific parameters involved in accuracy for gaze information (Symons et al., 2004). By using a gaze-contingent design in the development of our stimuli, we were guaranteed a certain measure of control during stimulus generation. This particular method can be adapted for even tighter control than that used in this experiment and may be useful in future experiments exploring gaze perception itself-specifically, in the ability to know, at any given time during stimulus generation, where the model fixated in relation to the target and the amplitude, speed, and accuracy of the saccade. For this reason, we have offered our database of 1,512 gaze stimuli to the research community.

\section{Conclusion}

The present work sought to exploit human sensitivity to gaze for the practical purpose of using a web camera to determine the locus of a participant's gaze. Such a system has the ability to allow for the detection and discrimination of eye movements as small as $1^{\circ}$. Considering that with eye movements up to $3^{\circ}$, detection and discrimination performance is around $90 \%$, it is clear that this system can be used as a reliable tool both for ensuring that individuals are maintaining fixation and for determining where their eyes are looking.

Acknowledgements This work was supported by Natural Sciences and Engineering Research Council of Canada (NSERC) operating grants to AK and NSERC and Killam Postdoctoral Fellowships to EFR.

Author Note The authors would like to thank Craig Anderson, Kaitlin Laidlaw, Jennifer Risko, Robert Teszka, and the Brain and Attention Research Lab team for their invaluable help on this project.

\section{References}

Anders, G. (2001). Pilot's attention allocation during approach and landing eye- and head-tracking research in an A330 full flight simulator.In Proceedings of the 11th International Symposium on Aviation Psychology. Retrieved August 9th, 2010, from http://www. faa.gov/library/online_libraries/aerospace_medicine/sd/media/ Anders.pdf

Anstis, S. M., Mayhew, J. W., \& Morely, T. (1969). The perception of where a face or television "portrait" is looking. The American Journal of Psychology, 82, 474-489.

Bellard, F. (2007). FFmpeg. Retrieved from http://ffmpeg.org/

Birmingham, E., Bischof, W. F., \& Kingstone, A. (2008). Gaze selection in complex social scenes. Visual Cognition, 16, 341-355.

Bock, S. W., Dicke, P., \& Thier, P. (2008). How precise is gaze following in humans? Vision Research, 48, 946-957.

Brennan, A.A., Watson, M.R., Kingstone, A., \& Enns, J.T. (2010, May). Third-person perception informs first-person visual search in a natural setting. Poster session presented at the annual meeting of the Association for Psychological Science, Boston, MA. 
Chisholm, J.D., Risko, E.F., \& Kingstone, A. (2010, August). Embodying attentional states: The role of posture in task performance. Poster presented at the Cognitive Science Societyannual meeting, Portland, OR.

Cline, M. G. (1967). The perception of where a person is looking. The American Journal of Psychology, 80, 41-50.

Duchowski, A. (2002). A breadth-first survey of eye-tracking applications. Behavior Research Methods, Instruments, \& Computers, 34, 455-470.

Dunai, J., Bennett, K., Fotiades, A., Kritikos, A., \& Castiello, U. (1999). Modulation of unilateral neglect as a function of direction of object motion. NeuroReport, 10, 1041-1047.

Fantz, R. L. (1965). Visual perception from birth as shown by pattern selectivity. Annals of the New York Academy of Sciences, 118, 793-814.

Fernald, A., Zangl, R., Portillo, A.L., \& Marchman, V.A. (2008). Looking while listening: Using eye movements to monitor spoken language comprehension by infants and young children. In I. Sekerina, E. M. Fernández, \& H. Clahsen(Eds.).Developmental psycholinguistics: On-line methods in children's language processing(pp. 97-135). Amsterdam: Benjamins.

Findlay, J. M., \& Walker, R. (1999). A model of saccade generation based on parallel processing and competitive inhibition. Behavioural \& Brain Sciences, 22, 661-721.

Gibson, B. S., \& Kingstone, A. (2006). Visual attention and the semantics of space: Beyond central and peripheral cues. Psychological Science, 17, 626-627.

Gibson, J. J., \& Pick, A. D. (1963). Perception of another person's looking behaviour. The American Journal of Psychology, 76, 386-394.

Hansen, D. W., \& Pece, A. E. C. (2005). Eye tracking in the wild. Computer Vision and Image Understanding, 98, 155-181.

Henderson, J. M., \& Hollingworth, A. (1998). Eye movements during scene viewing: An overview. In G. Underwood (Ed.), Eye guidance in reading and scene perception (pp. 269-294). Amsterdam: Elsevier.

Hutchins, E. (1995). Cognition in the wild. Cambridge, MA: MIT Press.

Kingstone, A., \& Pratt, J. (1999). Inhibition of return is composed of attentional and oculomotor processes. Perception \& Psychophysics, 61, 1046-1054.

Kingstone, A., Smilek, D., \& Eastwood, J. D. (2008). Cognitive ethology: A new approach for studying human cognition. British Journal of Psychology, 99, 317-340.

Kobayashi, H., \& Kohshima, S. (2001). Unique morphology of the human eye and its adaptive meaning: Comparative studies on external morphology of the primate eye. Journal of Human Evolution, 40, 419-435.

Land, M. F., \& Lee, D. N. (1994). Where we look when we steer. Nature, 369, 742-744.

Leigh, J. R., \& Zee, D. S. (2006). The neurology of eye movements (4th ed.). New York: Oxford University Press.

Li, D., \& Parkhurst, D.J. (2006).Open-source software for real-time visible-spectrum eye tracking. In Proceedings of the COGAIN Conference(pp. 18-20). Turin, Italy.

Macmillan, N. A., \& Creelman, C. D. (1991). Signal detection theory: A user's guide. Cambridge: Cambridge University Press.
Martinez-Conde, S., Macknik, S. L., \& Hubel, D. H. (2004). The role of fixational eye movements in visual perception. Nature Reviews. Neuroscience, 5, 229-240.

Masson, M. E., \& Loftus, G. R. (2003). Using confidence intervals for graphically based data interpretation. Canadian Journal of Experimental Psychology, 57, 203-220.

Pedersen, B.,\& Spivey, M. (2006). Offline tracking of eyes and more with a simple Webcam. In Proceedings of the 28th Annual Meeting of the Cognitive Science Society.

Posner, M. I. (1980). Orienting of attention. The Quarterly Journal of Experimental Psychology, 32, 3-25.

Pratt, J., Kingstone, A., \& Khoe, W. (1997). Motor-based versus attention-based theories of inhibition of return. Perception \& Psychophysics, 59, 964-971.

Rayner, K. (1998). Eye movements in reading and information processing: 20 years of research. Psychological Bulletin, 124, $372-422$.

Richardson, D. C., \& Spivey, M. J. (2004). Eye tracking: Research areas and applications. In G. Wnek \& G. Bowlin (Eds.), Encyclopedia of biomaterials and biomedical engineering (pp. 573-582). New York: Taylor \& Francis.

Risko, E. F., \& Kingstone, A. (2011). Eyes wide shut: Implied social presence, eye tracking and attention. Attention, Perception, and Psychophysics, 73, 291-296.

Risko, E. F., Stolz, J. A., \& Besner, D. (2010). Spatial attention modulates feature crosstalk in visual word processing. Attention, Perception, \& Psychophysics, 72, 989-998.

Schreij, D., Owens, C., \& Theeuwes, J. (2008). Abrupt onsets capture attention independent of top-down control settings. Perception \& Psychophysics, 70, 208-218.

Snodderly, D. M., Kagan, I., \& Gur, M. (2001). Selective activation of visual cortex neurons by fixational eye movements: Implications for neural coding. Visual Neuroscience, 18, 259-277.

Spelke, E. S., Breinlinger, K., Macomber, J., \& Jacobson, K. (1992). Origins of knowledge. Psychological Review, 99, 605-632.

Symons, L. A., Lee, K., Cedrone, C. C., \& Nishimura, M. (2004). What are you looking at? Acuity for triadic eye gaze. The Journal of General Psychology, 131, 451-469.

Theeuwes, J., Kramer, A. F., Hahn, S., \& Irwin, D. E. (1998) Our eyes do not always go where we want them to go: Capture of the eyes by new objects. Psychological Science, 9, 379-385.

Vivero, V., Barriera, N., Penedo, M. G., Cabrero, D., \& Remeseiro, B. (2010). Directional gaze analysis in webcam video sequences. In A. Campilho \& M. Kamel (Eds.), Lecture notes in computer science: Vol. 6112. Image analysis and recognition (pp. 316-324). Berlin: Springer Berlin/ Heidelbert.

Wang, J. G., \& Sung, E. (2001). Gaze determination via images of irises. Image and Vision Computing, 19, 891-911.

Wedel, M., \& Pieters, R. (2000). Eye fixations on advertisements and memory for brands: A model and findings. Marketing Science, 19, 297-312.

Wolfe, J. M. (2010). Visual search. Current Biology, 20, R346-R349. 